\title{
Female-to-male transsexuals and gay-sounding voices: A pilot study
}

\author{
Lal Zimman \\ University of Colorado
}

\begin{abstract}
A great deal of work has now been published on the perception of men's sexual orientation on the basis of phonetic characteristics. In this paper, I present a pilot study focusing on a population that sheds new light on this topic: female-to-male transsexuals. As individuals who were raised as girls but self-identify as men, trans men (as they are also called) are often perceived as gay-sounding after undergoing the drop in vocal pitch that is typically brought on by testosterone therapy. Using recordings of read speech from three trans men and five non-trans men who were each rated as gay- or straight-sounding by listener subjects, the analysis presented here shows that trans men are perceived in much the same way as gay-sounding non-trans men, despite a number of differences in the acoustic features of their voices. Ultimately these findings lend credence to the notion that there is no single gay-sounding phonetic style, but rather multiple styles that are lumped together perceptually as gay-sounding on the basis of their deviation from norms for straight-sounding voices.
\end{abstract}

\section{Introduction}

As the study of language and sexuality has become an established subdiscipline within sociolinguistics over the past two decades, a number of linguists have taken an interest in the question of whether sexual orientation can be detected on the basis of particular phonetic features or styles, particularly among male speakers (Gaudio 1994; Levon 2007; Linville 1998; Munson, Jefferson and McDonald 2006; Munson et al. 2006; Munson 2007; Pierrehumbert, et al. 2004; Podesva, Roberts and Campbell-Kibler 2001; Podesva 2007; Smyth and Rogers 2002; and Smyth, Jacobs and Rogers 2003). ${ }^{1}$ While one of the most basic questions explored in this literature has been whether listeners can accurately judge male speakers as gay or straight based on voice alone, these authors have also sought to uncover the precise phonetic features that correlate with the perception of a man's voice as "gay-sounding."

This paper is a revised version of the author's preliminary examination for the $\mathrm{PhD}$ in Linguistics at the University of Colorado, Boulder. Thanks are owed to Rebecca Scarborough for her help in designing this research, and to Steven Duman, Joshua Raclaw, Richard Sandoval, Susanne Stadlbauer, and an anonymous CRIL reviewer for feedback during the revision of this work.

1 A few studies (Waksler 2001; Pierrehumbert et al. 2004; Munson et al. 2006; Munson, Jefferson \& McDonald 2006) have also examined the voices of lesbian women and/or women perceived as lesbian-sounding. The findings of these studies are important, but beyond the scope of the present paper.

Colorado Research in Linguistics. June 2010. Vol. 22. Boulder: University of Colorado.

(C) 2010 by Lal Zimman. 
In this paper, I focus on these same perceptual and acoustic questions, but I also provide new insight through the introduction of a group of speakers that are almost completely absent from the sociocultural linguistic literature: female-tomale transsexuals. Female-to-male transsexuals, who are also called trans men, are individuals who are assigned to a female gender role and raised as girls, but who come to identify as men at some point later in life. Trans men make for an especially interesting community for sociophonetic study because of their unique set of experiences with the biological and socialized aspects of the voice. While trans men typically have female-sounding voices before their shift from a female social role to a male one, these individuals generally come to be heard as malesounding over the course of their gender role transition. This is in large part due to the fact that many trans men make use of testosterone for hormone replacement therapy, which results in a marked drop in vocal pitch just as it does during typical male puberty. In fact, as I show in later sections of this paper, trans men's voices are indistinguishable from the voices of other men when it comes to fundamental frequency. On the other hand, many of the differences between men's and women's voices have been shown to be learned during childhood rather than determined by the biological differentiation that arises during adolescence. And since trans men are expected to grow up as girls and later become women, their experiences with socialization are different from those had by men who are raised as boys.

The questions driving the research described in this paper have to do with the consequences of this mixture of biological and social factors that is characteristic among trans men. Because some authors studying gay-sounding voices have suggested that boys who acquire "feminine" phonetic traits during childhood might come to sound gay as adults (Smyth and Rogers 2002; Renn 2002), my goal in undertaking this work has been to explore whether trans men would be described as gay-sounding by listeners in a perceptual experiment like those conducted by other authors. If trans men's voices do indeed tend to be gaysounding, I also aim to discover which vocal features might explain this perception, and whether the acoustic characteristics of trans men's voices are the same as those found among gay-sounding non-trans men.

I begin this paper with an overview of the findings of previous studies on gay-sounding men's voices, which provide a starting point for my own analysis. Notably, the findings of these studies have often been contradictory, making it difficult to construct a unified model of gay-sounding men's voices. Rather than presenting a challenge to be overcome, however, I argue that the differences in these findings likely reflect real-life diversity among gay-sounding speakers. In other words, different studies have reached different conclusions because there are in fact multiple phonetic styles that might be interpreted as gay-sounding. In section 3, I discuss my own research comparing the voices of trans men to the voices of both gay-sounding and straight-sounding non-trans men. What my findings show is that trans men are indeed perceived as gay-sounding: members of this group were rated by listeners in the same way as the gay-sounding non- 
trans men. However, my phonetic analysis reveals a number of differences between these groups when it comes to acoustic measurements. In the discussion section I ultimately argue that these findings support the idea, introduced by Zwicky (1997), that different phonetic styles are lumped together as gay-sounding by listeners simply because they deviate from a straight-sounding norm. I conclude with reflections on how the linguistic practices of trans men can enhance our understanding of gay-sounding voices more generally, and with directions for future research.

\section{Previous research}

A number of studies, such as those carried out by Gaudio (1994), Linville (1998), and Smyth, Jacobs and Rogers (2003), have compared speakers' selfidentified sexual orientation to listeners' perception of these same individuals as straight or gay on the basis of read speech. Consistently, such work has shown that listeners are able to identify speakers' sexual orientations at better than chance rates. At the same time, however, each of these authors have noted that the correlations they have uncovered are imperfect, simply because not all gay men sound gay and a few straight men do. Nevertheless, this work suggests there is a salient socio-perceptual category for "gay-sounding" voices. Having shown that such a grouping exists on the perceptual level, these authors and others have focused on uncovering the acoustic characteristics that correlate with the categorization of a particular voice as gay- or straight-sounding.

As I mentioned in the previous section, it is difficult to synthesize the findings of research on gay-sounding voices because of the way different studies have sometimes reached contradictory conclusions. For example, most research that has investigated speakers' mean fundamental frequency have shown no difference between gay- and straight-sounding men on this measure (Gaudio 1994; Linville 1998; Podesva, Roberts and Campbell-Kibler 2001; Smyth and Rogers 2002). However, Munson et al.'s (2006) study, which analyzed words produced in isolation rather than connected speech, found that the gay-sounding men in their study did have higher fundamental frequency than the straightsounding men. The same study also found that gay-sounding men had higher mean F1 and F2 than straight-sounding men, with Munson (2007) further confirming the significance of mean F1. However, all of the other studies that have compared mean F1 and F2 across gay- and straight-sounding speakers show no significant differences (Linville 1998, Smyth and Rogers, 2002; Pierrehumbert et al. 2004). Vowel duration also seems to play some role in the perception of men's sexual orientation, but it isn't clear whether this difference is found only in certain vowels (Podesva, Roberts and Campbell-Kibler 2001; Smyth and Rogers 2002), in all vowels (Munson et al. 2006), or potentially not at all (Pierrehumbert et al. 2004). Crucially, whether a variable correlates with listeners' perception of a voice as gay-sounding seems to depend in part on the other variables present in 
the speakers' style: Levon (2007) found that decreasing a gay speaker's pitch range through digital manipulation lead to reduced gayness ratings by listeners, but increasing a straight speaker's pitch range had no significant effect on how his voice was rated.

Some of the variation that shows up in these findings is no doubt due to differences in data collection methods, which have included individual words (Munson Jefferson and McDonald 2006; Munson et al. 2006; Munson 2007) and connected read speech (Gaudio 1994; Linville 1998; Pierrehumbert et al. 2004; Smyth and Rogers 2002) and spontaneous speech (Smyth, Jacobs and Rogers 2003) produced in laboratory conditions, as well as an unscripted radio broadcast (Podesva, Roberts and Campbell-Kibler 2001). However, there is another very important potential explanation, which is that speakers perceived as gay-sounding are probably not all using the same phonetic style. As I mentioned in the previous section, this is the argument advanced by Zwicky (1997). Specifically, he says that there is unlikely to be a single set of characteristics that can be delineated as the gay-sounding style (also see Podesva, Roberts and Campbell-Kibler 2001 for a highly nuanced treatment of this idea). Instead, as Zwicky suggests, virtually any deviation from ways of talking associated with heterosexual masculinity whether by virtue of a higher pitch, higher first and second formants, greater duration for vowels and/or consonants, or any of the other of numerous features that have been investigated - can be interpreted as indexing gay identity. This idea is highly intuitive, given the pervasive cultural discourse that equates any kind of gender non-normativity, particularly among men, with homosexuality (discussed in detail by Gaudio 1994). There are also empirical findings to support Zwicky's argument. Gordon (2008) presents an analysis that compares the same speakers delivering gay-sounding and straight-sounding readings of the same passage. Gordon found that speakers made use of a wide variety of styles in their gay-sounding guises, each characterized by different marked phonetic variants, while their straight-sounding guises were much more similar to one another's. What these speakers' gay-sounding readings had in common, then, was their deviation from a more homogenous straight-sounding style.

Although authors have often reached different conclusions regarding which acoustic features are salient in the perception of sexual orientation, many researchers working on this topic have pointed out the similarities between the speech of gay-sounding men and that of women. Particularly in the work of Smyth and his colleagues (especially Smyth and Rogers 2002), gay-sounding voices have been characterized as a mixture of features typically associated with men's voices, such as a relatively low mean F0, with other features typically associated with women's voices, such as relatively longer sibilants or vowels that are articulated closer to the periphery of the vowel space. Given these similarities, some authors have suggested that men who reject or fail to conform to heteronormative masculinity are more apt to sound gay than men with more conventional and ideologically unmarked enactments of gender (Renn 2002; Smyth and Rogers 2002). More specifically, gender socialization during 
childhood is presented in this work as a likely source for gay-sounding voices among men, given that children are known to acquire sociophonetic markers of gender quite early in life (e.g. Sachs 1975). For instance, Smyth and Rogers (2002) argue that despite getting similar linguistic input from adults, some boys may engage in selective intake by orienting more strongly to women speakers as linguistic role models rather than men. ${ }^{2}$ Considering what we know about the acquisition of other forms of sociolinguistic distinction (such as regional dialect) and the way that adolescents negotiate their role in the heterosexual marketplace as part of a peer-based social order (Eckert 2003), young speakers' age cohorts probably also play a considerable role in the socialization of gendered phonetic traits.

Based on the arguments presented by these authors, it is worth considering the possibility that men who grow up orienting to the norms for women speakers in their communities, rather than men, will tend to be judged as gay-sounding in adulthood. The present study addresses this question from a rather unusual angle: by focusing on the voices of trans men. As I mentioned in the introduction, trans men very often make use of testosterone therapy as part of their transition from a female gender role to a male one, which generally results in a great deal of physiological masculinization, including changes in the larynx. One study of trans men's voices, which appears to be the only of its kind (described in both van Borsel et al. 2000 and in Adler and van Borsel 2006), found that the two individuals studied experienced a significant decrease in mean F0 and in F0 range, which put them within a normative male range during the first year of testosterone therapy. On the other hand, testosterone has no apparent effect on the many phonetic cues for speaker gender that are learned during language socialization, such as differences in segment duration or vowel quality (see Simpson 2009 for a review). Of course, given that trans men are raised in a female gender role, their experiences with childhood language socialization are markedly different from most men's. If trans men do differ from most other men in terms of socially-learned gendered phonetic traits, and if these speakers are perceived as gay-sounding men, then the unique experiences of members of this group would seem to provide evidence that childhood gender socialization can be a significant factor in predicting whether a man will be perceived as gay- or straight-sounding, at least for some speakers.

In order to explore this issue, the remainder of this paper is devoted to a comparison of men from three groups: trans men (hereafter TM), non-trans men with gay-sounding voices (GSM), and non-trans men with straight sounding voices (SSM). In this space I focus on two questions: first, how are the voices of TM perceived, compared to GSM and SSM? Second, what are some of the acoustic similarities and differences between members of these groups? Before

2 Of course, it might just as easily be the straight-sounding boys who are engaging in selective intake by orienting only to men rather than also paying attention to women, or that all children are engaging in some kind of selective intake in choosing their speaker role-models. 
answering these questions, section 3 will describe the methods for data collection and analysis used in this project.

\section{Methods}

\subsection{Data collection}

In order to test the questions presented above, speakers were recruited through previous research contacts and the researcher's own extended social network at the University of Colorado and in San Francisco, CA. None of the participants were aware of the topic of the investigation before being recorded beyond the fact that they were participating in a study about "how men talk." Three speakers were recorded from each of the three groups under investigation: TM (trans men), GSM (gay-sounding non-trans men), and SSM (straightsounding non-trans men). The GSM and SSM speakers were initially selected on the basis of whether I perceived them to be gay-sounding or straight-sounding, but these perceptions were then checked against listener ratings (see section 3.2 below). Speakers were from urban or suburban areas in the Western US and were between the age of 20 and 27, with the exception of one 47 year old speaker in the GSM group. While the non-trans men each identified as either gay or straight, all of the trans men identified with broader and potentially more fluid sexuality labels, such as bisexual, pansexual, and/or queer.

Following the methodology described by Smyth, Jacobs and Rogers (2003), speakers were recorded while reading two passages: the rainbow passage (Fairbanks 1960), which is an historical and scientific overview of rainbows, and the fire passage (Crist 1997), which is a dramatic narrative about a building fire. However, the final analysis presented in this paper includes only the fire passage (the text of this passage can be found in Appendix A). This choice was motivated primarily by Smyth, Jacobs and Rogers' finding that the scientific rainbow passage tended to evoke inflated gayness ratings for speakers who were perceived as straight in other contexts. Additionally, these authors found no significant differences between the dramatic read passage and a spontaneous spoken passage, suggesting that the dramatic passage is more representative of speakers' more naturalistic speaking styles. ${ }^{3}$ In addition, technical problems with the computer used for recording meant that a few speakers had to reread the scientific passage, which had a clearly audible effect on the speed at which they read; obviously, this would problematize the comparison of segment duration. While read speech is known to differ from naturally-occurring discourse in a number of ways and thus limits the generalizability of this study, read speech was chosen to facilitate the perceptual experiment described in section 3.2 as well as providing easily

\footnotetext{
${ }^{3}$ Two volunteers for this study also pointed out the symbolic significance of rainbows in the gay community. Although Smyth, Jacobs and Rogers assume that genre is the only factor at work here, it could be that the topic also influences listeners' judgments or even speakers' production.
} 
comparable data for acoustic analysis. However, these findings should be seen as a starting point that will guide future studies that make use of interactional language data (see section 5). Recordings were made with a Samson C03U USB multi-pattern condenser microphone and digitized at $48,000 \mathrm{~Hz}$ using the Audacity recording software program (Audacity Development Team 2008).

\subsection{Listener evaluation}

Identical segments of approximately 30 seconds were extracted from each speaker's reading of the fire passage. Eight listener subjects, all native speakers of American English, were then recruited to evaluate these clips via an online survey. The survey presented each audio clip along with sets of binary adjectives from which listeners were instructed to choose; for example, they were asked to rate how tall or short each speaker sounded on a scale of 1 to $5 .{ }^{4}$

Listeners were instructed to play the audio clips and mark each speaker's characteristics according to their best guess, but there was also an option to choose "no clue" to signify that the listener had no guess whatsoever as to a particular characteristic. Listeners were not instructed on the purpose of the experiment, nor that collecting the gay versus straight ratings were the primary purpose of the study. Discussion of the other social characteristics listeners rated is beyond the scope of the present analysis.

On the basis of listener perceptions, one speaker from the GSM group was excluded - despite my perception of him as gay-sounding, listeners consistently perceived him as a straight-sounding speaker (i.e. his gayness ratings were not significantly different from the straight men in this study). Because the goal was to compare the voices of straight-sounding straight men and gay-sounding gay men (rather than defining groups primarily on self-identification), only 2 speakers from the GSM group were included for analysis.

\subsection{Acoustic analysis}

Each thirty second clip that was played for listener subjects was also subjected to acoustic analysis using the Praat software package (Boersma and Weenink 2008). The features chosen for this analysis were selected on the basis of previous studies' findings and included the following measures:

1. Voiceless sibilant consonants (20 tokens of /s/, 1 token of $/ \mathrm{s} /$ )

a. Mean duration

b. Mean frequency at peak amplitude

c. Mean center of gravity

\footnotetext{
${ }^{4}$ Other traits, in addition to gay versus straight and masculine versus feminine, included young
} versus old, rude versus polite, and short versus tall. 
2. Vowels
a. Mean F0 across 11 stressed vowels
b. F0 range across 11 stressed vowels
c. Mean F1 and F2 across 11 stressed vowels
d. F1 and F2 of /æ/ (3 stressed tokens) and / $/ \mathcal{E} /(2$ stressed tokens)

Given the stereotype of the "lisping" gay man, it is unsurprising that researchers of gay-sounding voices have often directed their attention to sibilant consonants. Indeed, variation in the properties of $/ \mathrm{s} /$ has been consistently shown to correlate with the perception of men's voices as gay- or straight-sounding. Many researchers have investigated the duration of these sounds (Linville 1998; Podesva, Roberts and Campbell-Kibler 2001; Smyth and Rogers 2002; though see also Levon 2006, 2007), but some have also focused on the acoustic qualities of these segments themselves (Linville 1998; Munson et al. 2006; Munson 2007). Sibilant consonants, like other fricatives, are characterized by high-frequency aperiodic energy. Fricatives can be distinguished from one another by which frequencies are most prominent in terms of amplitude. That is, while /s/ tends to have relatively high-amplitude energy at around $8,000 \mathrm{~Hz}$, the highest-amplitude energy in $/ \mathrm{S} /$ tends to be closer to 4,000 Hz (Johnson 1997:130). Thus, one measure that has been used in investigations of sibilants in general, and in gaysounding sibilants in particular, has been the frequency of the sound at peak amplitude (Linville 1998). Similar information can be gathered through the measurement of the center of gravity of $/ \mathrm{s} /$, which provides a holistic view of which frequencies have the highest amplitude within a sound. Another measure that has received attention is spectral skew (Munson et al. 2006; Munson 2007), which refers to whether the majority of acoustic energy is located in the higher frequencies of the sound or the lower frequencies, but this particular measure was not used in the present study. In order to compare the sibilant consonants of the speakers in this study, I identified the instances of $/ \mathrm{s} /(n=20)$ and $/ \mathrm{J} /(n=1)$ in the thirty second clips played for listeners. I then measured each token's length, generated a spectral slice for the token, ${ }^{5}$ from which the peak frequency was identified visually, and finally generated center of gravity measurements using Praat's automated moments analysis function, which were checked against visual examinations of the spectra. Because the 20 tokens of /s/ appeared in identical phonemic contexts, comparisons across speakers used the mean values of these measurements (i.e. mean duration, mean center of gravity, etc.).

Vowel quality has also been consistently shown to influence the perception of sexual orientation. Although men with gay-sounding voices have not usually been found to have overall higher mean formants than straight sounding men, some research has turned up differences in the quality of individual vowels in terms of either F1 or F2 (Smyth and Rogers 2002;

5 A spectral slice provides a visual representation of the relationship between frequency and amplitude within a sound at a given point in time. 
Pierrehumbert et al. 2004; Munson et al. 2006). In this study, I discuss two of these vowels: $/ \mathfrak{x} /$ and $/ \varepsilon /$. I analyzed three stressed instances of $/ \mathfrak{x} /$ and two stressed instances of $/ \mathcal{E} /$ from each 30 -second sample. In addition to the five tokens of $/ \mathfrak{x} /$ and $/ \varepsilon /$, six other stressed vowels were included in the analysis in order to have a broader range of data from which to calculate F0 measurements, including three tokens of $/ \mathrm{i} /$, two tokens of $/ \mathrm{a} /$, and a single token of $/ \mathrm{\Lambda} /$. Each of these vowels was measured for duration; maximum, minimum, and mean F0 across the entire token; mean F1 and F2 across the entire token; and F1 and F2 at two points in the vowel, approximately $1 / 3$ and $2 / 3$ of the way through the segment (estimated visually).

\section{Results}

\subsection{Perceptual results}

Before discussing the acoustic findings of this project, it is important to establish which speakers were perceived as gay-sounding and which were perceived as straight-sounding. Aside from the GSM speaker who was eliminated from the sample (see section 3.2 above), listener evaluations correlated strongly with my preliminary groupings of men as gay- or straight-sounding (see Appendix B for listener ratings). A one-way ANOVA test ${ }^{6}$ showed that speakers' average numerical rating for gayness interacted significantly with the group in which they had been placed (SSM, GSM or TM). The group effects, as calculated by a post hoc (Tukey HSD) test, can be seen in Table 1 .

Table 1: Speaker grouping vs. gayness rating

\begin{tabular}{cc} 
Group comparison & P-values \\
\hline SSM vs. GSM & $0.0171 *$ \\
SSM vs. TM & $0.0313^{*}$ \\
GSM vs. TM & 0.5994 \\
$*$ * significant at .05
\end{tabular}

As the table shows, there was a highly significant difference between the gayness ratings given to the speakers in the SSM and GSM groups, confirming

${ }^{6}$ Statistical analyses were performed using the R Project for Statistical Computing software (R Development Core Team 2008). ANOVA is a useful approach to the data in question because it allows for the comparison of data on a linear continuum instead of requiring the use of categorical variables like traditional statistical software for sociolinguistic analysis (i.e. VARBRUL). 
that listeners shared my perception of the GSM speakers as gay-sounding and the SSM speakers as straight-sounding. Notably, there was also a significant difference between the gayness ratings for speakers in the SSM and TM groups. However, there was no significant difference between the GSM and TM speakers.

We are now in a position to answer one of the questions driving this research: How are the voices of trans men perceived, compared to gay-sounding and straight-sounding men? These perceptual data indicate that TM and GSM speakers are both perceived as significantly more gay-sounding than the SSM speakers. Furthermore, the fact that there is no significant difference between the gayness ratings given to the GSM and TM groups suggests that these groups are lumped together perceptually as gay-sounding men, in contrast to the straightsounding speakers in this study. ${ }^{7}$ However, the second question to be addressed in this paper remains: are the voices of GSM and TM as similar acoustically as they are perceptually?

\subsection{Sibilants}

As I discussed in section 3, one set of measurements I took was of the voiceless sibilant consonants $/ \mathrm{s} /$ and $/ \mathrm{J} /$, including duration, frequency at peak amplitude, and center of gravity. Beginning with /s/, I calculated each speaker's mean for duration, peak frequency, and center of gravity across 20 tokens. I then ran statistical tests that compared each of these means against three factors: first, speaker group (e.g. do speakers in the GSM and/or TM category have a longer mean duration for /s/ length than those in the SSM group?); second, gayness rating (do speakers who were rated by listeners as more gay-sounding have a higher peak frequency than those who were rated as less gay-sounding?); and finally, masculinity rating (do speakers who were rated by listeners as less masculine have a higher center of gravity than those who were rated as more masculine?).

Based on these tests, the only statistically significant interaction was between group and center of gravity $(p<0.0012)$. Specifically, the TM group had a significantly higher center of gravity in the distribution of energy in $/ \mathrm{s} /$ than either the SSM or GSM groups. The specific group interactions, as shown by post hoc analysis, can be seen in table 2 . These results show that while there was no statistically significant difference between SSM and GSM speakers, there were significant differences between both the SSM and TM groups and between the GSM and TM groups. In this case, then, the voices of TM and GSM are not alike acoustically, despite their similarity perceptually.

While the interaction of center of gravity and group was the only statistically significant result from this set of measurements, a few other results

${ }^{7}$ Listeners were explicitly told that all speakers were male in order to avoid the possibility that some speakers might be perceived as female. 
were statistically suggestive (i.e. with p-values less than .1), namely, both the interaction between masculinity rating and sibilant duration $(p<0.0947)$ and the interaction between masculinity rating and peak frequency $(p<0.0766)$ approached significance. Both of these factors thus deserve ongoing attention in future extensions of this research.

Table 2: Speaker grouping vs. center of gravity for $/ \mathrm{s} /$

\begin{tabular}{cc} 
Group comparison & P-values \\
\hline SSM vs. GSM & 0.1152 \\
SSM vs. TM & $0.0043^{*}$ \\
GSM vs. TM & $0.0012 *$ \\
$*$ = significant at .05
\end{tabular}

Although there was only one token of $/ \mathrm{S} /$ for comparison, center of gravity again provided a statistically significant result. However, in this case it is the GSM group that stands apart from the other two rather than the TM group.

Table 3: Speaker grouping vs. center of gravity for $/ \mathrm{J} /$

\begin{tabular}{cc} 
Group comparison & P-values \\
\hline SSM vs. GSM & 0.1040 \\
SSM vs. TM & 0.3384 \\
GSM vs. TM & $0.0234 *$ \\
$*=$ significant at .05
\end{tabular}

As table 3 shows, there is a significant difference between GSM and TM groups, and a difference that is nearly statistically suggestive between SSM and GSM groups, but no significant or suggestive difference between TM and SSM groups. Again, the TM and GSM groups are acoustically different, despite being perceptually similar. Interestingly, however, in this case it is the TM speakers who are like SSM speakers, whereas for /s/ it was the GSM group that resembled the SSM group. Also of interest is the fact that the GSM's center of gravity was significantly lower than the other two groups, when we might expect it to be higher. 


\subsection{Fundamental frequency}

Pitch was examined through measurements of fundamental frequency, but no significant or suggestive results were obtained. Neither mean F0 nor F0 range correlated with speaker group, gayness rating, or masculinity rating. Tests were also run on the F0 values for each of the individual stressed vowels examined in section 3.4, and again no significant results were obtained. In fact, the variation within groups was obviously greater than the variation across groups - the best example of this is the fact that the TM group contained both the speaker with the highest mean F0 and the speaker with the lowest mean F0 of all participants in the study.

\subsection{Vowel formants}

Like a number of other studies of gay-sounding voices, the data I analyzed showed no significant differences in speakers' overall mean first and second formants. As Smyth and Rogers (2002) have pointed out, this is one of the ways in which gay-sounding men's voices are different from women's. Indeed, I found no differences between the mean vowel formants for gay- and straight-sounding speakers, and this held true for both the GSM group and the TM group.

However, as I mentioned above, a few studies have shown individual vowels to be especially likely to differ between gay- and straight-sounding speakers. The current analysis produced similar findings. As I mentioned in section 2.3, I focused on the vowel quality of 3 stressed tokens of $/ \mathfrak{x} /$ and 2 stressed tokens of $/ \varepsilon /$ that appeared in the spoken excerpt played for listener subjects. Instead of taking the mean formant values for these vowels, I compared

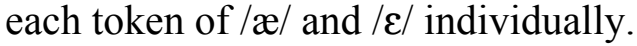

The first instance of stressed /æ/ appears in the sentence "they must have been trapped," (see Appendix A). For this token, a one-way ANOVA test showed that speakers' mean F2 for this vowel interacted significantly with speaker group $(p<0.0152)$. Specifically, the GSM group had a lower mean F2 than either the TM or SSM groups. The effects of each individual group, as shown by a post hoc test, are in Table 4. In this case there is again a significant difference between the GSM and TM speakers $(p<0.0286)$, further demonstrating that these groups are acoustically different even as they are perceptually similar. However, this is the only one of the three tokens of /æ/ that showed statistically significant interaction between F2 and speaker group (none showed significant interaction with gayness rating or masculinity rating), making this finding tentative until further analysis is carried out. One other token of $/ \mathfrak{x} /$ did show a statistically suggestive interaction between mean F1 across this vowel and both speaker gayness rating $(p<0.0972)$ 
and speaker masculinity rating $(p<0.0986)$. However, neither of the other two instances of /æ/ showed any significant or suggestive variation in F1 values.

Table 4: Speaker grouping vs. F2 for /al

\begin{tabular}{cc} 
Group comparison & P-values \\
\hline SSM vs. GSM & $0.0153 *$ \\
SSM vs. TM & 0.7619 \\
GSM vs. TM & $0.0285 *$ \\
& $*=$ significant at .05
\end{tabular}

The other vowel investigated in this analysis was $/ \varepsilon /$. Both instances of this vowel show a relationship between $\mathrm{F} 1$ and speaker gayness ratings as well as between F1 and speaker masculinity ratings. In the first token of $/ \varepsilon /$, which appears in the sentence, "but as soon as I poked my head out, I smelled smoke," there was a significant correlation between mean F1 for this vowel and gayness rating $(p<0.0432)$ as well as between $\mathrm{F} 1$ and masculinity rating $(p<0.0446)$. That is, speakers who were more gay-sounding (or less masculine-sounding) had relatively higher $\mathrm{F} 1$ values for $/ \mathcal{E} /$. The second token of this vowel, from the sentence, "the ambulance guys had to put a splint on his leg," showed the same pattern, but with only a statistically suggestive correlation between F1 and gayness rating $(p<0.0972)$ and between $\mathrm{F} 1$ and masculinity rating $(p<0.0986)$. The second token of $/ \varepsilon /$ also showed a statistically suggestive correlation between F2 and gayness rating $(p<0.0538)$ - specifically, speakers with higher gayness ratings had lower F2 values.

\subsection{Vowel duration}

Finally, vowel duration was examined, both as a mean across all stressed vowels analyzed for this project as well as individually within the stressed tokens of $/ \mathfrak{a} /$ and $/ \varepsilon /$ discussed above. Only one such comparison yielded statistically significant results, which was the duration of the second token of $/ \varepsilon /$. In this case a one-way ANOVA showed that the duration of this segment correlated significantly with speaker group $(p<0.0391)$ such that duration of this segment in the GSM group was significantly longer than it was for the SSM group. While the difference in duration was only statistically suggestive when comparing the GSM and TM groups, there was no statistical difference between the SSM and TM group, suggesting that TM are patterning more closely along the lines of the SSM 
speakers rather than the GSM speakers. The effects of each group can be seen in table 5.

Table 5: Speaker grouping vs. vowel duration (token \#1 of /E/)

\begin{tabular}{cc} 
Group comparison & P-values \\
\hline SSM vs. GSM & $0.0363 *$ \\
SSM vs. TM & 0.6755 \\
GSM vs. TM & 0.0849. \\
.$=$ & suggestive at .1, * = significant at .05
\end{tabular}

These results serve as a final illustration of the ways in which the voices of TM and GSM are not alike acoustically.

\subsection{Summary}

This section has described several significant differences across the three speaker groups under investigation. First, for sibilant consonants, speakers in the TM group have a higher center of gravity for /s/ than the GSM and SSM groups, while speakers in the GSM group have a lower center of gravity for $/ \mathrm{s} /$ than the TM and SSM groups. There may also be a connection between perceived masculinity and sibilant length and peak frequency. In terms of vowels, speakers in the GSM group had a significantly lower F2 for one instance of /æ/, but there were no differences in the two other examples. Additionally, speakers with higher gayness ratings had higher $\mathrm{F} 1$ values for $/ \mathcal{\varepsilon} /$; it is also possible that speakers with higher gayness ratings had lower F2 values for this vowel. Finally, speakers in the GSM group had a longer duration for the second token of $/ \varepsilon$ / than speakers in either the SSM or TM groups. In terms of mean F0, F0 range, and overall mean $\mathrm{F} 1$ and F2, there were no significant differences across these groups.

\section{Discussion}

The results just presented point to a very significant conclusion that also serves to answer the second research question of this paper: given that we have established (in section 4.1) that the speakers in the TM and GSM groups are both perceived as gay-sounding compared to the speakers in the SSM group, are these two sets of speakers' voices as similar acoustically as they are perceptually? 
Several findings in this study suggest that the answer is "no." While some of the phonetic variables I have discussed correlated with the speaker's mean gayness or masculinity rating regardless of group membership, suggesting that both TM and GSM speakers are using these features, other variables were used primarily by TM speakers or GSM speakers. Specifically, TM speakers had a higher center of gravity for $/ \mathrm{s} /$, while the GSM speakers had a lower center of gravity for $/ \mathrm{J} /$, a lower F2 for one instance of /æ/, and a longer duration for one token of $/ \varepsilon /$. These results thus provide support for Zwicky's suggestion that there is more than one kind of gay-sounding voice, and that any number of different kinds of male voices that differ significantly from the straight-sounding norm can be perceived as indexing gay identity.

The fact that trans men seem to be particularly likely to have gay-sounding voices even if they don't identify as gay also suggests that early life gender socialization may very well be an important factor in accounting for why some men have gay-sounding voices and others do not - and, especially, why some men who do not identify as gay might nevertheless sound gay. Of course, this isn't to say that gender socialization is the only factor at work here. The fact that the trans men in this study did not identify as straight and for the most part tended to reject mainstream limitations on masculinity is surely relevant as well. However, considering that phonetic gender differences in the very features discussed here are known to arise early in life (e.g. Flipsen et al. 1999 on /s/), socialization during childhood deserves more attention. At the same time, it isn't as simple as saying that men who were raised as girls must be somehow inherently more feminine than men who were raised as boys - gender socialization does not have the same effect on everyone. If gender socialization always "worked" to produce gender normative adults, transsexuals would probably not exist, nor would many other kinds of gender diversity. Why else would one trans speaker (\#2 in Appendix B) have such a high gayness rating at 4.167 out of 6 , while another trans speaker (\#4) had a much lower rating at 2.714 ? These two trans men are the same height, speak very similar varieties of American English, and are both queer-identified but have had little contact with communities of gay men. In fact, we might expect speaker \#4 to have the higher gayness rating, because he was in a long-term relationship with a gay man at the time of recording, while speaker \#2 has been in a long-term relationship with a straight woman for several years. Clearly, these issues of gender, socialization, sexual orientation, and the interaction between them deserve more theoretical development than has so far been applied to this literature. Unfortunately, a thorough exploration of these issues is outside the scope of the current project and will have to wait for future extensions of this work.

There are also a few limitations of the study described in this paper that provide good reason for building on this work in the future. First, the small number of speaker subjects, especially in the GSM group, is an obvious weakness. In addition to more speakers, future work will also include more 
features for acoustic analysis in order to achieve a fuller picture of how speakers talk. Vowels in particular will reveal a more comprehensive picture of how these speakers vary from one another if examined as a system rather than in isolation. One final limitation is that read laboratory speech does a relatively poor job of reflecting how people actually speak in interaction. While Smyth, Jacobs and Rogers (2003) found no significant difference between a spontaneous spoken passage and the dramatic passage used in this study, there is no reason to believe that even their spontaneous spoken passage produced in laboratory conditions would reflect a speaker's more typical manners of speaking in everyday interaction. The findings put forth by Podesva (2007) emphasize this point - he studied the use of falsetto as a stylistic resource for constructing a gay identity, but it is extremely unlikely that a speaker who makes use of falsetto during interaction with his friends, for example, would employ it while reading into a microphone, or even while engaged in a sociolinguistic interview. However, the results from this pilot will be highly valuable as a jumping off point for further work that makes use of interactional language data.

One issue in this research that might be perceived as a problem is the fact that only one token of $/ \mathfrak{x} /$, out of the three analyzed, showed statistically significant variance across speakers and only one token of $/ \varepsilon /$ showed significant differences in F2 and duration. However, this may simply be a reflection of normal intra-speaker variation - in other words, even the most gay-sounding speakers don't necessarily sound equally gay all of the time. It may be that pronouncing a single word in a way that sounds gay is sufficient to create the perception that the speaker is gay. ${ }^{8}$ It is also worth noting that both of these tokens appeared in sentence-final position, while the other instances of these vowels were mid-clause. Position in a syntactic or intonational phrase may thus play some part in determining which vowels are likely to be marked by this sort of sociolinguistic variation. Extending the scope of analysis and including a greater number of speakers and tokens would also likely aid in answering these questions more satisfactorily.

A final issue that may have complicated this analysis is the variability within the TM group. While these speakers were demographically similar (European-American, queer identified transsexual men in their early 20s of comparable physical size), the length of time since their transition varied considerably. One speaker (\#2) began living in a male social role and taking testosterone approximately eight years before this recording was made (starting at age 15), another (\#4) started testosterone approximately three years prior to being recorded (at age 19), and the last (\#5) had started testosterone only 8 months prior (at age 20). Mean F0 did correlate, among these speakers, with the length of time since they had started testosterone therapy. Speaker \#5, who began testosterone therapy at age 15, also had by far the lowest F0 among these speakers - indeed,

${ }^{8}$ See Mendoza-Denton 2008 for an example of this phenomenon among Latina gang members' use of Chicano English features. 
the lowest of any speaker in this study - and this may be related to the fact that beginning testosterone therapy at an early age is thought to have more dramatic effects. However, these findings also suggest that previous studies on transsexual men's voices (van Borsel et al. 2000; Adler \& van Borsel 2006) have been limited by only recording during the first year of testosterone therapy, as changes appear to continue beyond that point.

To extend this work, I am currently completing the data collection phase of a larger project that builds on this pilot. In addition to a larger number of speaker and listener subjects, the analysis also includes a greater variety of acoustic measures, including voice quality, overall vowel expansion, a greater number of individual vowel classes with larger numbers of tokens for each class, and other sociophonetic features implicated in the linguistic construction of gender and sexuality. Furthermore, a great deal more work is needed in order to explain, from a sociocultural linguistic perspective, why trans men tend to have gay-sounding voices and what this tells us about the indexical nature of gender and sexuality more generally.

\section{Conclusion}

In this paper, I have argued that the voices of trans men are perceived in much the same way as are the voices of gay-sounding non-trans men. However, there are important acoustic differences between these two groups in terms of both vowels and sibilant consonants. This supports a theory advanced by Zwicky (1997) over a decade ago but which has yet to be fully integrated into scholarship on gay-sounding voices: there is more than one kind of gay-sounding phonetic style. Further study is needed to confirm and expand on the results discussed in this paper, but the findings presented here are a promising starting ground for understanding the relationship between the many varieties of non-heteronormative voices.

\section{References}

Adler, Richard K. and John van Borsel. 2006. "Female-to-male considerations." In Richard K. Adler, Sandy Hirsch, and Michelle Mordaunt (eds.), Voice and Communication Therapy for the Transgender/Transsexual Client: A Comprehensive Clinical Guide, 139-167. San Diego: Plural Publishings.

Audacity Development Team. 2008. Audacity (Version 1.2.6.) [Computer program]. http://audacity.sourceforge.net.

Boersma, Paul and David Weenink. 2008. Praat: Doing phonetics by computer (Version 5.0.34) [Computer program]. http://www.praat.org. 
van Borsel, John, Griet de Cuypere, Robert Rubens, and B. Destaerke. 2000. "Voice problems in female-to-male transsexuals." International Journal of Language \& Communication Disorders 35(3): 427-442.

Crist, Sean. 1997. "Duration of onset consonants in gay male stereotyped speech." University of Pennsylvania Working Papers in Linguistics 4(3): 53-70.

Eckert, Penelope. 2003. "Language and gender in adolescence." In Miriam Meyerhoff \& Janet Holmes (eds.), Handbook of Language and Gender, 381400. Malden, MA: Blackwell.

Fairbanks, Grant. 1960. Voice and Articulation Drillbook. New York: Harper \& Row.

Flipsen, Peter, Jr., Lawrence Shriberg, Gary Weismer, Heather Karlsson and Jane McSweeny. 1999. Acoustic characteristics of /s/ in adolescents. Journal of Speech, Language, and Hearing Research 42(3): 663-677.

Gaudio, Rudolf P. 1994. "Sounding gay: Properties in the speech of gay and straight men." American Speech 69(1): 30-57.

Gordon, Bryan. 2008. "Gay sounds: A non-discrete model of gay speech." Paper presented at the Lavender Languages and Linguistics $X V$, Washington, D.C., February 18.

Johnson, Keith. 1997. Acoustic \& Auditory Phonetics. Malden, MA: Blackwell.

Levon, Erez. 2006. "Hearing 'gay': Prosody, interpretation, and the affective judgments of men's speech." American Speech 81(1): 56-78.

Levon, Erez. 2007. "Sexuality in context: Variation and the sociolinguistic perception of identity." Language in Society 36(4): 533-554.

Linville, Sue Ellen. 1998. "Acoustic correlates of perceived versus actual sexual orientation in men's speech." Folia Phoniatrica et Logopaedica 50(1): 35-48.

Mendoza-Denton, Norma. 2008. Homegirls: Language and Cultural Practice among Latina Youth Gangs. Malden, MA: Blackwell.

Munson, Benjamin, Sarah V. Jefferson, and Elizabeth C. McDonald. 2006. "The influence of perceived sexual orientation on fricative identification." Journal of the Acoustical Society of America 119(4): 2427-2437.

Munson, Benjamin, Elizabeth C. McDonald, Nancy L. DeBoe and Aubrey R. White. 2006. "Acoustic and perceptual bases of judgments of women and men's sexual orientation from read speech." Journal of Phonetics 34(2): 202240.

Munson, Benjamin. 2007. "The acoustic correlates of perceived masculinity, perceived femininity, and perceived sexual orientation." Language and Speech 50(1): 125-142.

Pierrehumbert, Janet B., Tessa Bent, Benjamin Munson, Ann R. Bradlow and J. Michael Bailey. 2004. "The influence of sexual orientation on vowel production." Journal of the Acoustical Society of America 116(4): 1905-1908.

Podesva, Robert J. 2007. "Phonation type as a stylistic variable: The use of falsetto in constructing a persona." Journal of Sociolinguistics 11(4): 478-504.

Podesva, Robert J., Sara J. Roberts, and Kathryn Campbell-Kibler. 2001. "Sharing resources and indexing meanings in the production of gay styles." In 
Kathryn Campbell-Kibler, Robert J. Podesva, Sarah J. Roberts, and Andrew Wong (eds.), Language and Sexuality: Contesting Meaning in Theory and Practice, 175-189. Stanford, CA: CSLI Publications.

R Development Core Team. 2008. "R: A Language and Environment for Statistical Computing (Version 2.7.2)" [Computer program]. Vienna, Austria: R Foundation for Statistical Computing. http://www.r-project.org.

Renn, Peter. 2002. "Subtypes of male homosexuality: Speech, male sexual orientation, and childhood gender nonconformity." Unpublished BA Thesis, University of Texas at Austin.

Sachs, Jacqueline. 1975. "Cues to the identification of sex in children's speech." In Barrie Thorne and Nancy Henley (eds.), Language and Sex: Difference and Dominance, 152-171. Newbury, MA: Newbury House Publishers.

Simpson, Adrian P. 2009. "Phonetic differences between male and female speech." Language and Linguistics Compass 3(2): 621-640.

Smyth, Ron and Henry Rogers. 2002. "Phonetics, gender, and sexual orientation." Proceedings of the Annual Meeting of the Canadian Linguistic Association, 299-311. Montreal, Canada: L’Universite du Quebec au Montreal.

Smyth, Ron, Greg Jacobs and Henry Rogers. 2003. "Male voices and perceived sexual orientation: An experimental and theoretical approach." Language in Society 32(3): 329-350.

Waksler, Rachelle. 2001. "Pitch range and women's sexual orientation." Word 52(1): 69-77.

Zwicky, Arnold. 1997. "Two lavender issues for linguists." In Anna Livia and Kira Hall (eds.), Queerly Phrased: Language, Gender, and Sexuality, 21-34. New York: Oxford University Press. 


\section{Appendix A}

The following is the passage from Crist (1997) that was read by speakers. The italicized portion is the segment that was played for listeners and analyzed:

You wouldn't believe what just happened! I was just sitting here studying, and it was getting pretty late, and I was going to go to bed here pretty soon. But then I started hearing these people screaming out in the street. So I got up, and I was going to yell out the window, "Will you please hold it down out there!" But as soon as I poked my head out, I smelled smoke, and you know that ski store down at the end of the corner? It was all full of flames. There were all these people in the apartments upstairs screaming out of the windows; they must have been trapped. I was scared that the fire might spread down the street to my place too. Then I heard sirens screaming, and all these cop cars and fire trucks pulled up. The firemen went up on ladders and helped all the people get out. One girl looked like she had bad burns on her skin, and this other guy fell, and the ambulance guys had to put a splint on his leg. I could see the guys down on the ground; they were having some kind of problem with the fire hydrant, but they finally got the hoses hooked up to the spouts, and then they went up and poked a hole in the roof with a big metal kind of stick, and they sprayed tons and tons of water in. It took them better than two hours to get the fire out. You know that Spanish student down the hall from me? Later, he told me he heard the owner set the fire himself. The whole thing was a big scam to get the insurance money. Unbelievable! 


\section{Appendix B}

The following table includes the mean ratings assigned to each speaker subject on a scale of straight to gay $(1=$ definitely straight, $5=$ definitely gay $)$ and masculine to feminine $(1=$ definitely masculine, $5=$ definitely feminine $)$.

\begin{tabular}{cccc} 
Speaker \# & Speaker group & Man gayness & Mean masculinity \\
\hline 2 & TM & 4.167 & 4.167 \\
4 & TM & 2.714 & 2.857 \\
5 & TM & 3.33 & 3.5 \\
3 & SSM & 2 & 2 \\
7 & SSM & 2 & 2.429 \\
8 & SSM & 1.4 & 1.4 \\
9 & GSM & 4.125 & 4 \\
10 & GSM & 3.666 & 4
\end{tabular}

